\title{
Rotating Optical Attenuator for the Generation of Subsecond Duration Sawtooth Shape Radiance Pulses*
}

\author{
Ared Cezairliyan \\ Institute for Materials Research, National Bureau of Standards, Washington, D.C. 20234
}

(August 28, 1970)

\begin{abstract}
A rotating attenuator for the generation of subsecond duration sawtooth shape radiance pulses is described. The attenuator disk is in the form of a cam. The geometry of the opening of a diaphragm used as the aperture stop determines the shape of the radiance pulses. Radiance is determined from the measurements with a high-speed photoelectric pyrometer. Recording of signals is made with a high-speed digital data acquisition system. The combined measuring and recording systems have a full-scale signal resolution of approximately one part in 8000 and a time resolution of $0.4 \mathrm{~ms}$. Two different diaphragms are used in this study yielding radiance pulses (20 to $150 \mathrm{~ms}$ long) with linear and quadratic rise. The standard deviation of the experimental points from the pertinent functions describing the radiance variation is less than 0.5 percent.
\end{abstract}

Key words: High-speed methods; light attenuator; photometry; radiance modulation; radiation.

\section{Introduction}

A considerable amount of work has been done to develop various techniques to modulate radiance beams in the visible as well as in the ultraviolet and infrared regions. Most of these techniques were confined to generating either repetitive rectangular pulses, or shortduration fast-risetime single pulses. A review of various methods for modulating radiance pulses was given by Jones [1]. ${ }^{1}$ Optical modulation techniques in the infrared region were discussed by Hudson [2].

Increasing interest in transient techniques for the measurement of properties of matter at high temperatures and related studies has necessitated the development of programmable optical attenuators. Such attenuators are needed to simulate (using steady-state radiance sources) the rapid heating or cooling of the specimen and also to check dynamic characteristics of radiation detectors and of high-speed temperature measurement systems in general.

\section{Method and Apparatus}

In this study an optical system, including a rotating optical attenuator, is described that is capable of generating repetitive sawtooth shape (either linear or quadratic) radiance pulses, ranging from $20 \mathrm{~ms}$ to several seconds in duration. The optical system (fig. 1) consists of a radiance source (tungsten filament lamp), a rotating disk (cam with helical circumference), a special diaphragm, a high-speed pyrometer, and other pertinent optical components. The lenses 01 and 02 shown in figure 1 are 63 $\mathrm{mm}$ in diameter with an aperture of $f / 2.5$. The circumference of the disk was machined in such a way that its

* This work was supported in part by the Propulsion Division of the U.S. Air Force Office of Scientific Research under contract ISSA-69-0001.

1 Figures in brackets indicate the literature references at the end of this paper. radius $r$ was proportional to the angle from a fixed reference point according to the following relation:

$$
r=r_{0}-k a
$$

where $r_{0}$ is the maximum radius, $\alpha$ is the angle, and $k$ is the proportionality constant. The maximum and minimum radii of the disk were 7 in $(177.8 \mathrm{~mm})$ and 6 in $(152.4$ $\mathrm{mm})$, respectively, yielding a maximum difference of 1 in $(25.4 \mathrm{~mm})$. The thickness of the disk was 0.12 in $(3 \mathrm{~mm})$.

The aperture stop of the optical system was defined by the circumference of the disk and the diaphragm placed in the collimated portion of the beam. As the disk rotated, the effective cross-sectional area of the beam changed, which, in turn, generated sawtooth shape pulses. The shape of the diaphragm opening determined the shape of the radiance pulse. For example, a rectangular opening as seen in figure 2 yields a pulse with linear rise, while a triangular opening gives a pulse with quadratic rise. Rotation of the disk was synchronized with other measuring equipment with a signal obtained from a radiation detector. The radiation detector PM (fig. 1) generated a signal each time a $0.2 \mathrm{~mm}$ in diameter hole $\mathrm{H}$ (fig. 2) fabricated in the disk was in line with the detector and the light source L (fig. 1). Relative radiance of the source after attenuation was measured with a high-speed photoelectric pyrometer [3]. The electrical signals from the pyrometer were recorded with a high-speed digital data acquisition system [4]. The combined measuring and recording system has a full-scale signal resolution of approximately one part in 8000 and a time resolution of $0.4 \mathrm{~ms}$.

The photomultiplier in the pyrometer was alternately exposed to the radiance from the tungsten filament lamp and to the radiance from a reference lamp. This scheme 


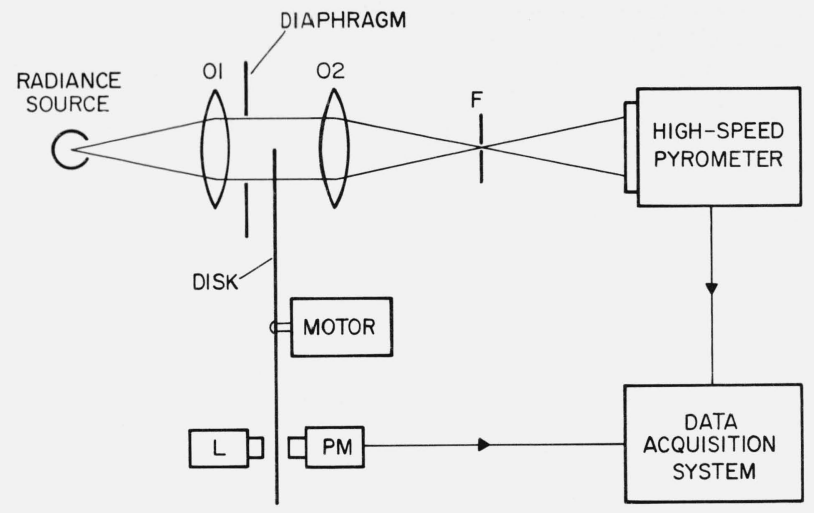

Figure 1. Functional diagram of the optical system for the generation of subsecond duration sawtooth shape radiance pulses.

eliminated errors that result from photomultiplier fatigue, etc.

Pulse shape as a function of time for various diaphragm geometries is derived in the following paragraphs.

The effective cross-sectional area of the light beam, when the disk circumference is at point $x$ on the diaphragm, may be expressed as

$$
A_{x}=\int_{0}^{x} y d x
$$

where $x=r_{0}-r$ and $y$ is the width (height) of the diaphragm at $x$ (fig. 2).
For rectangular geometry, $y=$ constant $=a$, thus

$$
A_{x}=a x \text {. }
$$

For triangular geometry, $y=b x$, thus

$$
A_{x}=b x^{2} / 2 \text {. }
$$

When the disk rotates at a constant speed, the angle of rotation is proportional to time $t$, that is

$$
a=k^{\prime} t \text {. }
$$

From eq (1) and the definition of $x$, it follows that

$$
x=r_{0}-r=k a .
$$

Combination of eqs (5) and (6) yields

$$
x=k k^{\prime} t \text {. }
$$

The radiance $L$ as seen by the pyrometer at a given instant is

$$
L=k^{\prime \prime} A \text {. }
$$

Combination of eqs (3), (4), (7), and (8) yields the following:

For rectangular geometry

$$
L=K t
$$

For triangular geometry

$$
L=K^{\prime} t^{2}
$$

where $K$ and $K^{\prime}$ are combination of constants. Equations (9) and (10) represent radiances that are linear and quadratic functions of time, respectively.

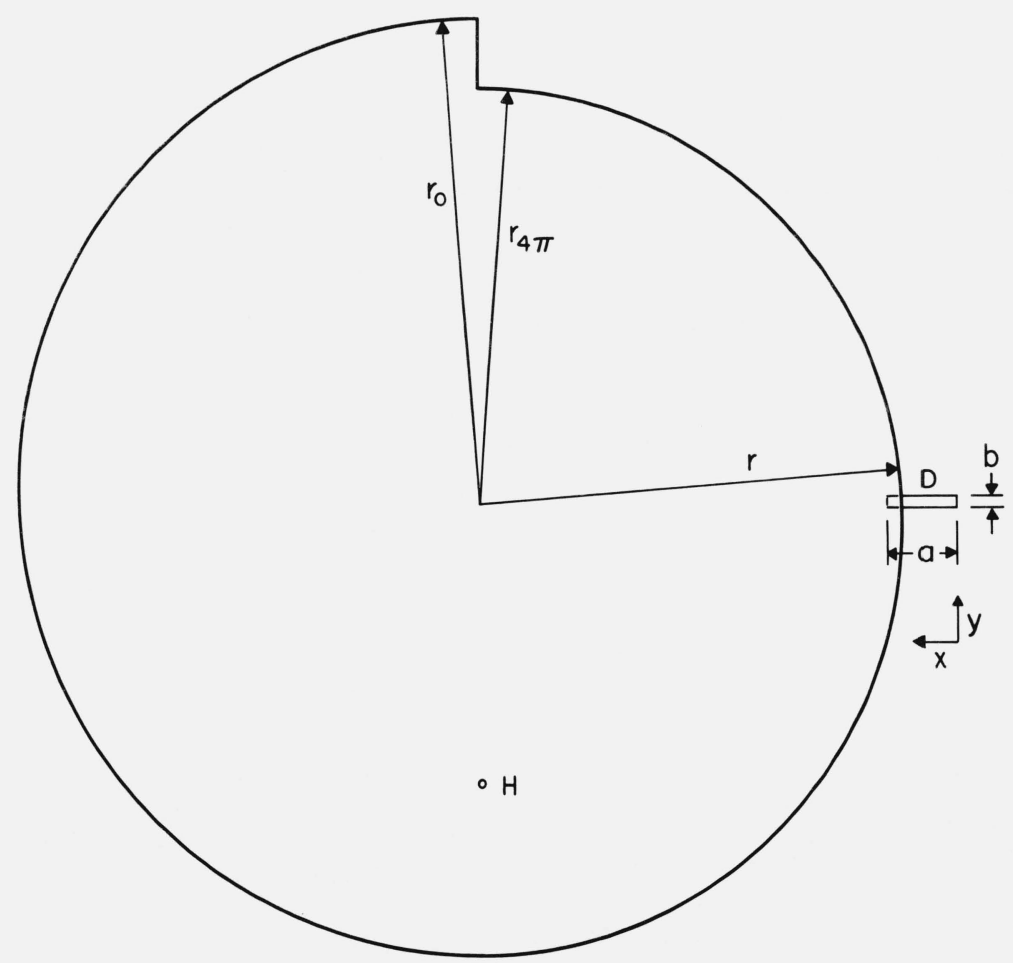

Figure 2. The attenuator disk and the diaphragm opening. 


\section{Experimental Results}

In order to assess the operational characteristics of the system, experiments were performed using diaphragms of rectangular and triangular openings. These experiments are summarized in the following paragraphs.

Linear radiance pulses: A total of 15 experiments were conducted at five different attenuator speeds yielding radiance pulses (increasing linearly with time) in the range 20 to $150 \mathrm{~ms}$. Additionally, four experiments were performed with the direction of rotation of the attenuator reversed. This scheme generated radiance pulses that decreased linearly with time.

Quadratic radiance pulses: A total of four experiments were conducted at two different attenuator speeds. An additional experiment was performed with the direction of rotation of the attenuator reversed.

Oscilloscope trace photographs of representative radiance pulses are shown in figure 3 . The radiances as detected by the high-speed pyrometer and recorded with the digital data acquisition system were fitted to linear and quadratic functions, depending on the pulse shape, using the least squares method. A summary of the experimental parameters and results is given in table 1. It may be seen that average standard deviation (individual point) of the results of both pulse shapes is approximately 0.6 percent corresponding to data covering a radiance ratio of over 10.

The above value for the standard deviation reflects departure from ideal behavior that may be due to several factors, such as random noise in radiance samples and in the pyrometer operation, nonuniformity in the lenses and in the optical alinement, nonuniformity in the attenuator circumference, variation in the attenuator speed, etc. In order to separate the contribution of the pyrometer and the random noise inherent in radiance measurements from the attenuator and the associated optical system, two separate experiments were conducted under steadystate conditions. In one of the experiments, the attenuator was positioned to provide the maximum opening of the diaphragm as seen by the pyrometer. Then the pyrometer response to the radiance source was recorded. The standard deviation of the results was 0.2 percent. A similar experiment was performed with the attenuator positioned to provide radiance equivalent to that of the lowest level

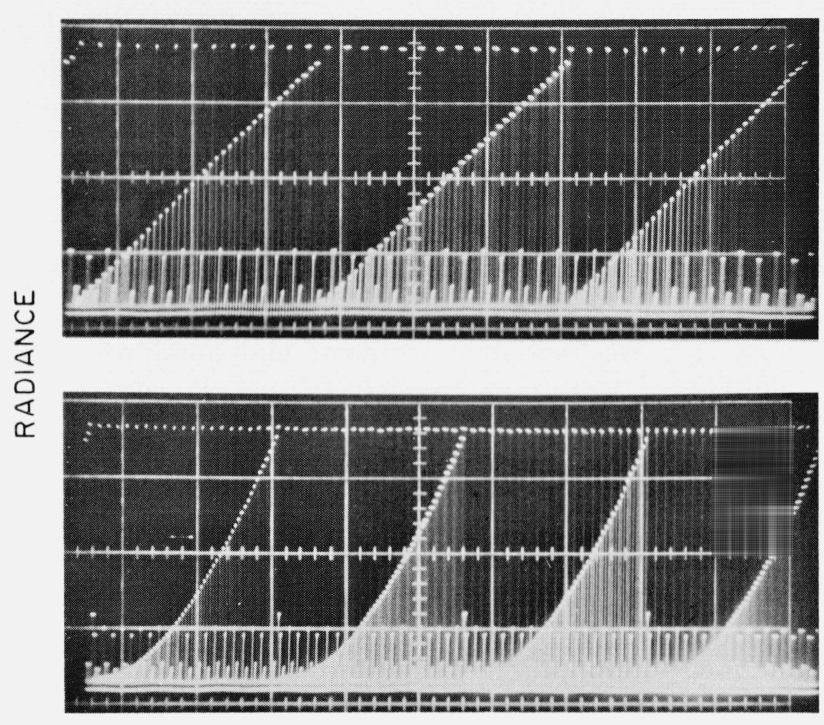

TIME

Figure 3. Oscilloscope trace photographs of linear (upper) and quadratic (lower) radiance pulses.

Horizontal scale: $10 \mathrm{~ms}$ (upper), $20 \mathrm{~ms}$ (lower) per major division. Vertical scale: arbitrary units. Dots forming the long horizontal lines correspond to radiances from the reference source.

used in the dynamic experiments. The standard deviation of the results of the pyrometer output was 0.8 percent. Assuming an approximately linear variation of randomness between the two extremes, a value of 0.5 percent for the standard deviation contributed by the pyrometer and radiance measurements in general is obtained. Thus, if it is assumed that total standard deviation is the square root of the sum of the squares of individual standard deviations, the standard deviation corresponding to the nonuniformities of the attenuator and the associated optical components becomes approximately 0.3 percent. This value is comparable to the uncertainty with which the pyrometer linearity could be determined in separate experiments.

In order to ascertain the fact that the value obtained for the standard deviation was the result of random deviations in the data, the data on radiance were also fitted

TABLE 1. Summary of experimental parameters and results

\begin{tabular}{|c|c|c|c|c|c|c|c|}
\hline Item & $\begin{array}{l}\text { Radiance } \\
\text { variation }\end{array}$ & $\begin{array}{l}\text { Direction of } \\
\text { radiance } \\
\text { variation }\end{array}$ & $\begin{array}{l}\text { Pulse } \\
\text { length } \\
(m s)\end{array}$ & $\begin{array}{l}\text { Number of } \\
\text { experiments }\end{array}$ & $\begin{array}{l}\text { Number of } \\
\text { points per } \\
\text { experiment }\end{array}$ & $\begin{array}{l}\text { Average } \\
\text { radiance } \\
\quad \text { ratio }\end{array}$ & $\begin{array}{c}\text { Average total } \\
\text { standard } \\
\text { deviation, \% }\end{array}$ \\
\hline 1 & Linear & Increasing & 23 & 3 & 20 & 10.3 & 0.6 \\
\hline 2 & Linear & Increasing & 30 & 3 & 26 & 12.1 & .7 \\
\hline 3 & Linear & Increasing & 39 & 3 & 33 & 9.0 & .6 \\
\hline 4 & Linear & Increasing & 59 & 3 & 50 & 11.8 & .5 \\
\hline 5 & Linear & Increasing & 144 & 3 & 123 & 11.3 & .6 \\
\hline 6 & Linear & Decreasing & 23 & 1 & 20 & 10.0 & .7 \\
\hline 7 & Linear & Decreasing & 30 & 1 & 26 & 9.5 & .7 \\
\hline 8 & Linear & Decreasing & 39 & 1 & 33 & 10.4 & .6 \\
\hline 9 & Linear & Decreasing & 59 & 1 & 50 & 10.6 & .5 \\
\hline 10 & Quadratic & Increasing & 60 & 3 & 39 & 11.0 & 6 \\
\hline 11 & Quadratic & Increasing & 40 & 1 & 26 & 10.1 & .5 \\
\hline 12 & Quadratic & Decreasing & 60 & 1 & 39 & 11.1 & .6 \\
\hline
\end{tabular}


to functions of one and two higher degrees than the pertinent one. No improvement in the standard deviation was observed, indicating that there was no detectable systematic curvature imposed on the proper function.

\section{Conclusion}

The results of the experiments described above have shown the feasibility of generating subsecond duration pulses of prescribed shapes (linear and quadratic) with a standard deviation of less than 0.5 percent over a radiance ratio of 10 . The method allows one to insert appropriate diaphragms in the optical system to obtain radiance pulses of various shapes. For example, in addition to linear and quadratic, it is possible to generate pulses of cubic, exponential, and other shapes.
The scheme of generating radiance pulses allows one to detect the linearity of radiation detectors under dynamic conditions, and also provides the experimenter with means of simulating high-speed heating or cooling experiments.

\section{References}

[1] Jones, O. C., J. Sci. Instr. 41, 653 (1964) .

[2] Hudson, R. D., Infrared System Engineering (John Wiley \& Sons, Inc., New York, 1969), p. 235.

[3] Foley, G. M., Rev. Sci. Instr. 41, 827 (1970).

[4] Cezairliyan, A., Morse, M. S., Berman, H. A., and Beckett, C. W., J. Res. Nat. Bur. Stand. (U.S.), 74A (Phys. and Chem.), 65 (1970).

(Paper 75C1-311) 